\title{
Predictive Value of Malignancy Risk Indices for Ovarian Masses in Premenopausal and Postmenopausal Women
}

\author{
Sinem Ertas ${ }^{1 *}$, Fisun Vural ${ }^{1}$, Ertugrul Can Tufekci ${ }^{1}$, Ahmet Candost Ertas $^{2}$, \\ Gultekin Kose ${ }^{1}$, Nurettin Aka ${ }^{1}$
}

\begin{abstract}
Background: To evaluate the predictive role of a risk of malignancy index in discriminating between benign and malignant adnexal masses preoperatively. Materials and Methods: A total of 408 patients with adnexal masses managed surgically between January 2010 and February 2014 were included. The risk of malignancy indices (RMI) 1, 2, 3 and 4 were calculated using findings for ultrasonography, menopausal status, and CA125 levels. Histopathologic results were the end point. ROC analysis was used for the sensitivity and the specificity of the models. Results: Some $37.6 \%$ of the cases were malignant in the postmenopausal group while $7.9 \%$ were malignant in the premenopausal group. Pelvic pain was the most common complaint, and the majority of the cases were diagnosed at stage 3. The RMI 1,2,3 and 4 yielded percentage sensitivities of 76.1,79.1,76.1 and 76.1 and specificities of 91.5, 89.1,90.6, 88.6, respectively. RMI 1 was the most reliable test in the general population according to AUC levels and Kappa statistics. From ROC analysis results of post/ premenopausal women, the RMI 1 (cut off: 200) yielded sensitivities of 84.0/60.9 and specificities of 87.7/92.5. With RMI 2 they were 88.6/60.9 and 80.0/91.0, with RMI 3 84.0/ 60.9 and 87.7/91.8, and with RMI 4 (cut off:400) 81.8/47.8 and 83.6 /44.0. Although test performance of RMI methods were good in a general population and postmenopausal women, the RMI inter-agreement validity was only moderate or fair in premenopausal women. Conclusions: Our study confirms the effectiveness of RMI algorithms in postmenopausal women. However, more sensitive tests are needed for premenopausal women.
\end{abstract}

Keywords: Ovarian cancers - malignancy - malignancy risk index - RMI - adnexal masses

Asian Pac J Cancer Prev, 17 (4), 2177-2183

\section{Introduction}

Adnexal masses are a problem frequently encountered in gynecological practice. To differentiate the ovarian mass that is benign or malign could change clinical approach. According to data from the United States; each year about 300,000 women are hospitalized because of adnexal masses. 13-21 percent of these women have malign adnexal masses (Curtin et al., 1994; NIH Consensus, 1994). In women deaths; ovarian cancer is the fifth cancer type (Gibbs et al., 2010). According to the American Cancer Society data; more than 21000 new cases will be diagnosed in 2014-2015 and approximately 14000 women will die because of ovarian cancer (Siegel et al., 2014). In the premenopausal period, only $7-13 \%$ adnexal masses are malign, but unfortunately in the postmenopausal period $30-45 \%$ adnexal masses are malign. The 5-year survival rate is about $30 \%$ of patients diagnosed with advanced stage. Whereas in the cases diagnosed at an early stage, the 5-year survival rate is about $90 \%$ (Su et al., 2013). Thus, early diagnosis is important. However, due to nonspecific complaints, the majority of the cases are diagnosed at advanced stages.

In daily gynecology practice, ultrasonography is used widely in clinics and in asymptomatic patients who have adnexal masses can be frequently diagnosed. Patients who have ovarian cancer should be referred to well educated and experienced gynecological oncology surgeons. Because of inadequate cytoreductive surgery, patients mostly need a second surgery and this situation increases the morbidity (Elit et al., 2002). The successful cytoreductive surgery is an important prognostic factor in ovarian cancer survey (Eisenkop et al., 1991; Kehoe et al., 1994).

In gynecological malignancies; tumor markers have a crucial role in screening, monitoring of treatment, follow-up and also for predicting recurrence of the disease (Aggarwal et al., 2010). The serum CA 125 is one of the most widely used serum markers. CA 125 levels increases in only $50 \%$ of early stage ovarian cancer and $90 \%$ of late 
stage ovarian cancer. Moreover, elevated CA 125 levels may be observed in the variety of the conditions. Such as ascites, menstruation, endometriosis, pelvic inflammatory diseases, liver diseases, and other malignant conditions (pancreas, breast, lung, and colon) (Ozols et al., 2005).

A pelvic examination, ultrasonographic assessment and tumor markers are part of a standard evaluation for an adnexal mass preoperatively. Finding a screening and diagnostic method for ovarian cancer is challenging due to high mortality and insidious symptoms. Various combined methods for evaluating the patients' risks have been proposed. According to a single process, combined methods give more sensitive and accurate results. In 1990, Jacobs et, al. initially developed RMI 1 that is a simple scoring method based on menopausal status, ultrasound findings and serum CA 125 level (Jacobs et al., 1990). To increase sensitivity and specificity of this scoring system Tingulstad et al. developed RMI 2 in 1996 and then RMI 3 in 1999 (Tingulstad et al., 1996; Tingulstad et al.,1999). Lastly, Yamamoto et al. added the parameter of tumor size to RMI scores and developed RMI 4 in 2008 (Yamamoto et al., 2008).

American Congress of Obstetrics and Gynaecology and Society of Gynecologic Oncologists recommend using of clinical, demographic, laboratory and imaging features for triage of the patients with adnexal masses. National Institute of Clinical Excellence and Royal College of Obstetrics and Gynecology guidelines accept Risk of Malignancy Index as the best model. (ACOG Committee, 2002; RCOG guideline, green top 34,2010; NICE clinical guidelines CG122,2011). Studies showed until now; RMI scoring system has the advantage of rapid and exact triage of the patients. Nevertheless, the literature shows that different populations have different sensitivity and cut-off values. (Ashrafgangooei et al., 2011; Ong et al., 2013).

The aim of this study was to evaluate the ability of the four malignancy risk (RMI 1,2,3,4) indices to discriminate a benign from a malignant pelvic mass. Moreover, besides the cut-off levels that are mostly used and suggested by researchers, we attempted to find cut off levels for each of malignancy indices in premenopausal and postmenopausal women.

\section{Materials and Methods}

\section{Study design}

This study designed retrospectively in Gynecology and Obstetrics Department of Haydarpasa Numune Training and Education Hospital that is a tertiary referral research and education hospital in Istanbul. The local ethics committee of the hospital approved the study Clinical details of all adnexal masses were prospectively entered into a computer, which were retrieved for analysis, retrospectively. A total of 417 patients with adnexal masses that underwent surgery between 1 January 2010 and 14 February 2014 enrolled in the study. The exclusion criteria from the study were an adnexal mass with pregnancy and patients with unavailable data or absent detailed ultrasonographic findings. We included 408 cases with complete data. The histopathological diagnosis was considered as the end point for determining the outcomes.
Malignant and borderline tumors were staged according to the criteria of International Federation of Gynecology and Obstetrics (Prat et al., 2014). The primary measure outcomes were the predictive value of ultrasonographic scoring RMI 1, 2, 3 and 4 in each pathologically proven malignancy.

\section{Basal Laboratory and Ultrasonographic Evaluation}

All patients underwent preoperative laboratory evaluation of blood count, biochemistry, the serum CA 125 levels and gray-scale ultrasonography. [CA 125 levels were measured by using radioimmunoassay ( Architect Abbott i2000sr CMIA)]. Ultrasound imaging of the cases was performed transvaginally and abdominally by an expert radiologist in our hospital that used Mindray DC7 ultrasound device with $5 \mathrm{Mhz}$ convex abdominal and 8 Mhz vaginal probes. The characteristic appearance of masses (bilaterality, multilocularity of the adnexal mass, solid areas, ascites, intraabdominal metastatic lesions, papillary projections, cystic formation, septate thickness) recorded carefully.

\section{Risk of Malignancy Index Scoring 1,2,3,4}

RMI scoring mainly is based on ultrasonographic features, menopausal status, and CA 125 levels (Jacobs et al., 1990; Tingulstad et al., 1996; Tingulstad et al.,1999). RMI 1, 2,3 were calculated by using the formula "RMI= M x US x serum CA 125". (M: menopausal status, U: ultrasound score). All formulas have different values for $\mathrm{M}$ and U. In RMI 4 calculation, tumor size $(\mathrm{S})$ is also included in the formula. RMI 4=U x M x Ca 125 x S (Yamamoto et al., 2008). Table 1 presents the RMI calculations.

Ultrasonographic features

The ultrasonographic features of adnexal masses such as multilocularity, bilaterality, solid areas, ascites and the presence of an extra-ovarian tumor are used in scoring system (Ekerhovd et al., 2001). RMI 1 (If there is no ultrasonographic findings, score was 0 . If 1 of the US features are found, score will be 1 . If $\geq 2$ US features are found, the score is 3 ). RMI 2 ( If none or 1 of the US features are found, the score was 1 . If $\geq 2$ US features are found, the score is 4). RMI 3( If none or 1 of the US features are found, the score was 1 . If $\geq 2$ US features are found, the score is 3). RMI 4( If none or 1 of the US features are found, the score was 1 . If $\geq 2$ US features are found, the score is 4 . If the tumor size is $\geq 7 \mathrm{~cm}, S$ is equal to 2 .

\section{Statistical analysis}

Statistical Package for Social Sciences for Windows 18.0 program was used. Data were analyzed using descriptive statistical methods (mean, standard deviation, frequency.) Student's t-test was used for the comparison of quantitative data with the regular distribution. In the intergroup comparison of the parameters of a normal distribution, Mann-Whitney test was used. For the comparison of qualitative data; Mc Nemar test, kappa analysis, and diagnostic screening tests were used. For determining the best cut-off values to discriminate malign adnexal masses from benign adnexal masses; a receiver operating characteristics (ROC) curve was plotted for 
Predictive Values of Malignancy Risk Indices for Ovarian Masses in Premenopausal and Postmenopausal Women

each malignancy risk indices. The sensitivity, specificity, positive predictive value (PPV), negative predictive value (NPV) and diagnostic accuracy of four malignancy risk ratios (RMI 1,2,3,4) were calculated for each case. The probability value $\mathrm{p}<0.05$ was considered as statistically significant.

\section{Results}

All patients' age ranges from 14 to 87 . Among the study population, 291 of the patients were premenopausal (71.4\%), and 117 of the patients were postmenopausal (28.6\%). In the postmenopausal group, $37.6 \%$ of the cases were malignant while $7.9 \%$ of the cases were malignant in the premenopausal group. Out of 408 patients, 341 of the them had benign adnexal masses, 55 patients had malign adnexal masses, and 12 of the patients has the borderline ovarian tumor. Pelvic pain was the most frequent complaint of the patients. Table 2 shows the characteristics of benign and malign adnexal masses. According to this, malignant cases had significantly increased age, parity, menopausal status, platelet counts, and CA 125 levels $(\mathrm{p}=0.000)$. FIGO staging were borderline $(\mathrm{n}=12,2.9 \%)$, Stage I $(n=16,3.9 \%)$, Stage II $(n=7,1.7 \%)$, Stage III $(n=20$, $4.9 \%)$ and Stage IV $(n=12,2.9 \%)$.

The average age of patients with benign adnexal

Table 1. RMI 1,2,3 and 4 calculations (Jacobs et al., 1990; Tingulstad et al., 1996; Tingulstad et al., 1999; Yamamoto et al. 2008)

\begin{tabular}{lcccc}
\hline Parameters & RMI 1 & RMI 2 & RMI 3 & RMI 4 \\
\hline USG Score (U) & & & & \\
No feature & 0 & 1 & 1 & 1 \\
1 feature & 1 & 1 & 1 & 1 \\
$\geq 2$ features & 3 & 4 & 3 & 4 \\
Menopausal status (M) & & & & \\
Premenopausal & 1 & 1 & 1 & 1 \\
Postmenopausal & 3 & 4 & 3 & 4 \\
CA 125 (IU/ml) & value & value & value & value \\
Tumor Size (S) & & & & \\
$<7$ cm & - & - & - & 1 \\
$\geq 7$ cm & - & - & - & 2 \\
\hline
\end{tabular}

masses was $40.8 \pm 13.8$, and the mean age of patients with malign adnexal masses was $54.4 \pm 13.6$ years. Malignant masses tend to occur in advanced ages-parity and postmenopausal women $(\mathrm{p}<0.05)$. Both premenopausal and postmenopausal period patients mostly had the diagnosis of the malignant ovarian mass at Stage III. Table 2 presents the characteristic findings of premenopausal and postmenopausal adnexal masses. In general population, the most common benign ovarian tumors were endometrioma and functional cysts. The most common malign ovarian tumors were epithelial cancers.

\section{General population}

The RMI 1 (cutoff: 200) yielded the sensitivity of $76.1 \%$, specificity of $91.5 \%$ [AUC:0.937, CI:0.9-0.96], PPV (76.1\%), NPV (91.4\%) and an accuracy of $88.9 \%$. The RMI 2 (cut off:200) yielded the sensitivity $79.1 \%$ and specificity of $89.1 \%$ [AUC:0.935, CI:0.9-0.96] , PPV of $79.1 \%$, NPV of $88.1 \%$ and an accuracy of $87.2 \%$. The RMI 3 (cut off:200) formula had a sensitivity $76.1 \%$ and specificity of $90.6 \%$ [AUC:0.923, CI: 0.88-0.95], PPV

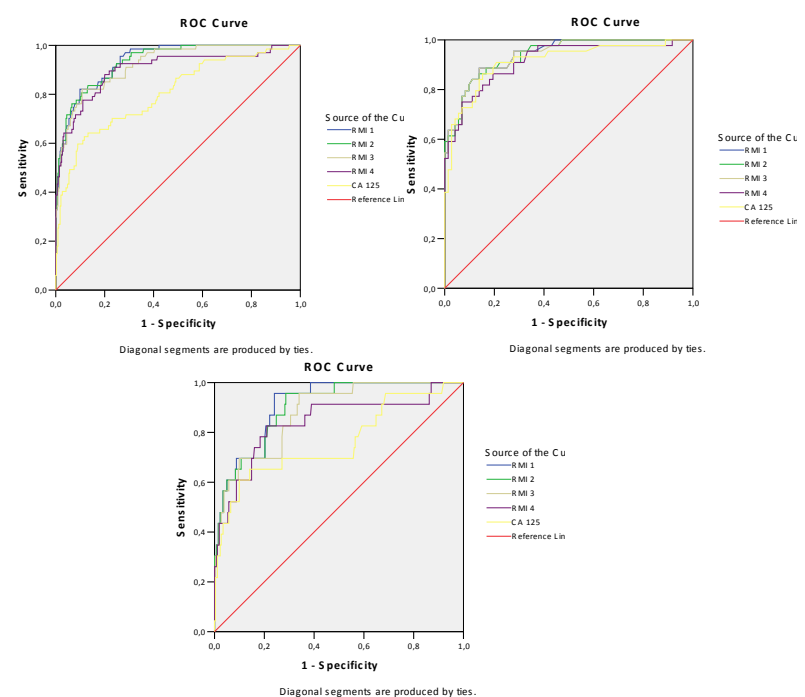

Figure 1. The ROC analysis of RMI 1, 2, 3, 4 and CA 125. a) general population; b) postmenopausal women; c) premenopausal women

Table 2. The characteristics of patients and laboratory evaluations of benign/malign and premenopausal/ postmenopausal adnexal masses

\begin{tabular}{|c|c|c|c|c|c|c|}
\hline Characteristic findings & Benign Masses & Malign Masses & $\mathrm{p}$ value & Premenopausal & Postmenopausal & $\mathrm{p}$ value 2 \\
\hline Patient characteristics & & & & & & 0.00 \\
\hline Age & $40.8 \pm 13.8$ & $54.4 \pm 13.6$ & 0.00 & $35.8 \pm 9.3$ & $61.1 \pm 8.9$ & 0.00 \\
\hline Gravidity & $2.9 \pm 2.6$ & $5.2 \pm 3.2$ & 0.182 & $2.2 \pm 2.0$ & $5.8 \pm 2.9$ & 0.00 \\
\hline Parity & $0.1 \pm 0.3$ & $0.05 \pm 0.2$ & 0.00 & $1.6 \pm 1.5$ & $4.5 \pm 2.7$ & 0.00 \\
\hline Abortion & $0.2 \pm 0.7$ & $0.5 \pm 1.2$ & 0.01 & $0.2 \pm 0.5$ & $0.5 \pm 1.2$ & 0.00 \\
\hline Menopausal status & $73 / 341(21.4 \%)$ & $44 / 67(65.6 \%)$ & 0.00 & $291 / 408(71.4 \%)$ & $\begin{array}{l}117 / 408 \\
(28.6 \%)\end{array}$ & 0.00 \\
\hline \multicolumn{7}{|l|}{ Laboratory Evaluation } \\
\hline Hematocrit (\%) & $36.6 \pm 4.2$ & $36.6 \pm 4.8$ & 0.979 & $36.3 \pm 3.9$ & $37.4 \pm 4.8$ & 0.01 \\
\hline Platelet (106/L) & $280000 \pm 83000$ & $330000 \pm 87990$ & 0.00 & $292000 \pm 85000$ & $278000 \pm 83000$ & 0.131 \\
\hline White Cell count (106/L) & $8819 \pm 3515$ & $8401 \pm 2677$ & 0.742 & $9227 \pm 10257$ & $7585 \pm 2040$ & 0.01 \\
\hline CA $125 \mathrm{U} / \mathrm{ml}$ & $63.5 \pm 218.5$ & $101.7 \pm 2506.7$ & 0.00 & $1.2 \pm 386$ & $3.7 \pm 1737.0$ & 0.121 \\
\hline CA $19-9 \mathrm{U} / \mathrm{ml}$ & $89.8 \pm 1145.2$ & $52.6 \pm 110.4$ & 0.810 & $1.0 \pm 1239$ & $70.9 \pm 425.8$ & 0.783 \\
\hline
\end{tabular}


of $76.1 \%$, NPV of $90.6 \%$ and an accuracy of $88.2 \%$. The RMI 4 (cut off:336) had a sensitivity $76.1 \%$ and specificity of $88.6 \%$ [AUC:0.903, CI: 0.85-0.94], PPV of $65.6 \%$, NPV of $92.3 \%$ and an accuracy of $87.9 \%$. The CA 125 levels had a sensitivity $56 \%$ and specificity $91.2 \%$, PPV of

Table 3. The results of ROC analysis and cutt off values of general population, postmenopausal and premenopausal women (sensitivity,specificity, AUC:area under curve, CI:confidence interval)

\begin{tabular}{|c|c|c|c|c|c|}
\hline Population & Sensitivity & Specificity & AUC & $\mathrm{p}$ value & $\mathrm{CI}$ \\
\hline \multicolumn{6}{|c|}{ General population } \\
\hline RMI 1 (200) & 76.1 & 91.5 & 0.937 & 0.00 & 0.90-0.96 \\
\hline RMI 2 (200) & 79.1 & 89.1 & 0.935 & 0.00 & $0.90-0.96$ \\
\hline RMI 3 (200) & 76.1 & 90.6 & 0.923 & 0.00 & $0.88-0.95$ \\
\hline RMI 4 (400) & 70.1 & 91.5 & 0.901 & 0.00 & $0.85-0.94$ \\
\hline RMI 4 (336) & 76.1 & 88.6 & 0.903 & 0.00 & $0.85-0.94$ \\
\hline CA125 (110) & 56.0 & 91.2 & 0.804 & 0.00 & $0.73-0.86$ \\
\hline \multicolumn{6}{|c|}{ Postmenopausal women } \\
\hline RMI 1 (200) & 84.0 & 87.7 & 0.940 & 0.00 & 0.89-0.97 \\
\hline RMI 2 (200) & 88.6 & 80.0 & 0.940 & 0.00 & 0.89-0.97 \\
\hline RMI 3 (200) & 84.0 & 87.7 & 0.939 & 0.00 & $0.89-0.97$ \\
\hline RMI 4 (400) & 81.8 & 83.6 & 0.915 & 0.00 & $0.85-0.96$ \\
\hline CA125 (50) & 68.2 & 93.2 & 0.914 & 0.00 & $0.85-0.96$ \\
\hline CA125 (110) & 54.0 & 97.3 & 0.914 & 0.00 & $0.85-0.96$ \\
\hline \multicolumn{6}{|c|}{ Premenopausal women } \\
\hline RMI 1 (200) & 60.9 & 92.5 & 0.911 & 0.00 & $0.85-0.95$ \\
\hline RMI 2 (200) & 60.9 & 91.0 & 0.901 & 0.00 & 0.84-0.95 \\
\hline RMI 3 (200) & 60.9 & 91.8 & 0.880 & 0.00 & $0.81-0.94$ \\
\hline RMI 4 (400) & 47.8 & 44.0 & 0.830 & 0.00 & $0.73-0.94$ \\
\hline RMI 4 (300) & 60.9 & 91.0 & 0.840 & 0.00 & $0.73-0.94$ \\
\hline CA125 (110) & 60.9 & 90.0 & 0.762 & 0.00 & $0.63-0.88$ \\
\hline
\end{tabular}

$56.7 \%$, NPV of $91.4 \%$ and an accuracy of $85.5 \%$ with cutoff value 110 . Table 3 shows the details. According to this, the RMI 1 was the most reliable in predicting malignancy in terms of area under the curves. The inter-agreement validity of tests was analyzed by Kappa statistics. The Kappa values were 0.627 for RMI 1, 0.594 for RMI 2, 0.609 for RMI 3. In the general population, RMI 1 test performance was good while others were moderate. If we exclude borderline cases from the malignant masses, the sensitivity of the test increased. The RMI 1 (cutoff:200) yielded the sensitivity of $83 \%$ and specificity of $90 \%$ in all age groups ( PPV:57\%, NPV:97\%, accuracy: 89\%). Figure 1a presents the ROC analysis of RMI 1, 2, 3, 4 calculations and CA 125 levels in general population.

\section{Postmenopausal women}

The RMI 1 at a cutoff level of 200 yielded the sensitivity of $84.0 \%$, the specificity of $87.7 \%$ [AUC:0.940, CI:0.89$0.97]$. The RMI 2 (cut off:200) yielded the sensitivity 88.6 $\%$ and specificity of $80.0 \%$ [AUC:0.940, CI:0.89-0.97]. The RMI 3 (cut off:200) formula had a sensitivity $84.0 \%$ and specificity of $87.7 \%$ [AUC:0.939, CI: 0.89-0.97]. The RMI 4 (cut off:400) had a sensitivity $81.8 \%$ and specificity of $83.6 \%$ [AUC:0.915, CI: 0.85-0.96]. The CA125 levels had a sensitivity $54 \%$ and specificity $97.3 \%$ with cut-off value 110. According to ROC analysis results, RMI 1,2,3 methods were similar according to area under the curve. The kappa values were 0.711 for RMI $1,0.667$ for RMI 2, 0.711 for RMI 3, and 0.619 for RMI 4. The RMI 1 and RMI 3 had better performance than RMI 2,4. Table 3 presents the details. Figure $1 \mathrm{~b}$ presents the ROC

Table 4. Review of the literature for RMI (cut off: 200 , *The cutoff value was 238 for RMI. **The cutoff value was 250 for $\mathbf{R M I}$ )

\begin{tabular}{|c|c|c|c|c|c|}
\hline Author & $\mathrm{n}$ & Sensitivity & Specificity & PPV & NPV \\
\hline Jacobs et al. (1990) & 143 & 85.4 & 96.9 & & \\
\hline Tingulstad et al. (1996) & 173 & 71.0 & 96.0 & 89.0 & 88.0 \\
\hline Tingulstad et al. (1999) & 365 & 71.0 & 92.0 & 69.0 & 92.0 \\
\hline Morgante et al. (1999) & 124 & 58.0 & 95.0 & 78.0 & 87.0 \\
\hline Manjunath et al. (2000) & 152 & 73.0 & 91.0 & 93.0 & 67.0 \\
\hline Andersen et al. (2003) & 180 & 70.6 & 87.7 & 66.1 & 89.8 \\
\hline Obeidat et al. (2004) & 100 & 90.0 & 89.0 & 96.0 & 78.0 \\
\hline Yazbek et al. (2006) & 106 & 89.0 & 92.0 & 50.0 & 99.0 \\
\hline Ulusoy et al. (2007) & 296 & 71.7 & 80.5 & 67.3 & 83.6 \\
\hline Van Trappen et al. (2007) & 174 & 76.0 & 82.0 & & \\
\hline Yamamoto et al. (2008) & 253 & 80.0 & 86.4 & 52.5 & 95.8 \\
\hline Clarke et al. (2009) & 163 & 72.0 & 87.0 & 75.0 & 85.0 \\
\hline Moolthiya et al. (2009) & 209 & 70.6 & 83.9 & 75.0 & 80.6 \\
\hline Van den Akker et al. (2010) & 548 & 81.0 & 85.0 & 48.0 & 96.0 \\
\hline Ashrafganggooei et al. (2011)* & 151 & 89.5 & 94.7 & 71.0 & 98.0 \\
\hline Aktürk et al. (2011) & 100 & 75.0 & 89.0 & 62.0 & 89.0 \\
\hline Van Gorp et al. (2012) & 432 & 76.0 & 92.0 & 87.0 & 85.0 \\
\hline Sayasneh et al. (2013) & 255 & 72.0 & 94.0 & & \\
\hline Terzic et al. (2013) & 540 & 83.8 & 77.2 & 47.0 & 98.1 \\
\hline Yavuzcan et al. (2013) & 153 & 75.0 & 90.1 & 66.7 & 93.2 \\
\hline Arun-Muthuvel et al. (2014) & 467 & 79.0 & 98.0 & 92.0 & 94.0 \\
\hline Ozun Ozbay et al. (2015)** & 191 & 60.0 & 93.0 & 75.0 & 88.0 \\
\hline Our study (2015) & 408 & 76.1 & 91.5 & 76.1 & 91.4 \\
\hline
\end{tabular}


Predictive Values of Malignancy Risk Indices for Ovarian Masses in Premenopausal and Postmenopausal Women

analysis of RMI 1, 2, 3, 4 calculations and CA 125 levels in postmenopausal women.

\section{Premenopausal women}

The RMI 1 at a cut-off level of 200 yielded the sensitivity of $60.9 \%$, specificity of $92.5 \%$ [AUC:0.91, CI:0.85-0.95]. The RMI 2 (cut off:200) yielded the sensitivity $60.9 \%$ and specificity of $91.0 \%$ [AUC:0.90, CI:0.84-0.95]. The RMI 3 (cut off:200) formula had a sensitivity $60.9 \%$ and specificity of $91.8 \%$ [AUC:0.88, CI: 0.81-0.94]. The RMI 4 (cut off: 400) had a sensitivity $47.8 \%$ and specificity of 44\% [ AUC:0.83, CI: 0.73-0.94]. The RMI 4 (cut off:300) had a sensitivity $60.9 \%$ and specificity of $91 \%$ [AUC:0.84,CI: 0.73-0.94]. The CA125 levels had a sensitivity $60.9 \%$ and specificity $90 \%$ with cut-off value 110. RMI 1 was the most reliable according to an area under the curve. The kappa values were 0.438 for RMI 1, 0.400 for RMI 2, 0.409 for RMI 3, and 0.364 for RMI 4. Table 3 presents the details. Figure 1c presents the ROC analysis of RMI 1, 2, 3, 4 calculations and CA 125 levels in premenopausal women.

\section{Discussion}

The risk of malignancy index algorithms is the most validated and accepted methods in discriminating benign and malign adnexal masses (RCOG guideline, green top 34,2010; NICE clinical guidelines CG122,2011). However, heterogeneity of the populations, diversity of ovarian tumors and prevalence lead different cut-off levels. This study evaluated the predictive value of four RMI calculations in pre and postmenopausal Turkish women. Our study confirms the effectiveness of RMI algorithms in postmenopausal women, but we still need tests with good performance in premenopausal women and borderline ovarian tumors.

Various diagnostic methods for adnexal masses have reported, such as abdominal and transvaginal ultrasonography, color Doppler ultrasonography, and tumor markers. However, none of these diagnostic methods used individually has shown significantly better performance in detecting early stage malignant tumors. The risk of malignancy index that include combined the risk of CA 125, menopause and ultrasonography, firstly developed by Jacobs et al. (1990). They found $85.4 \%$ sensitivity and the $96.9 \%$ specificity. They stated that women with RMI > 200 cut-off levels had 42 times risk of developing ovarian cancer. Subsequent to Jacobs' formula, Tingulstat et al. developed RMI 2 and RMI 3 respectively. Many papers published in this issue and different populations yield different results. Recently, Yamamoto et al. (2009) developed a new model for risk calculation of ovarian cancers. Table 4 presents the summary of the previous RMI studies.

The majority of the prior studies investigated the RMI values of the general population. Few studies explored these formulas in premenopausal and postmenopausal women (Terzic et al., 2013; Sayasneh et al., 2013; Kaijser et al., 2013). The sensitivity of any test is affected by the prevalence of the diseases. Our malignancy incidence in postmenopausal women $(37.6 \%)$ was similar to the other studies (30\%-43\%).(Jacobs et al., 1990; Kaijser et al., 2013). Since postmenopausal women have increased the risk of malignancy, the number of postmenopausal women affect the results. Apart from the prior studies, we explored RMI values in pre and postmenopausal Turkish women separately (Simsek et al., 2014; Yesilyurt et al., 2014; Akturk et al., 2011; Yavuzcan et., al 2013; Ozun Ozbay et al., 2015).

A systematic review study by Geomini et al., reviewed 109 studies including 21750 women with adnexal masses. RMI was the best predictor and when 200 were used as the cut-off level with $78 \%$ sensitivity and $87 \%$ specificity (Geomini et al., 2009). The RMI 1 studies from Turkey shows sensitivity varies from $60-76.1 \%$, and specificity varies between $88.8-93 \%$. Our results are in between them. We showed that RMI 1 (cut-off:200) yielded the sensitivity of $76.1 \%$, the specificity of $91.5 \%$. Kappa value for RMI 1 was 0.627 when the cut-off value was set at 200 for the general population. In our postmenopausal group, the kappa value was 0.711 the RMI 1 at a cut-off level of 200 yielded the sensitivity of $84.0 \%$, the specificity of $87.7 \%$. The premenopausal RMI 1 at a cut-off level of 200 yielded the sensitivity of $60.9 \%$, the specificity of $92.5 \%$ with kappa 0.438 . Thus, agreement of RMI 1 test in premenopausal women was moderate. The performance of RMI 1 in a general population and postmenopausal women was good.

In the study of Tingulastad et al. (1996) using the RMI 2 for the first time, 92\% specificity and $80 \%$ sensitivity were obtained with a cut-off value of 200 . In our general population, The RMI 2 (cut off:200) yielded the sensitivity $79.1 \%$ and specificity of $89.1 \%$ with kappa 0.594. Abdulrahman Jr. et al. studied the accuracy of RMI $1,2,3$ in the 247 Welsh women (Abdulrahman Jr.et al., 2014). RMI 2 (cut off:200) gave better performance than RMI 1 and 3. This finding was similar to prior reports (Tingulstad et al., 1996; Morgante et al., 1999; Moolthiya et al., 2009). In a comprehensive review, the pooled estimate of sensitivity was 79\% (71-78\%), and specificity was $81 \%$ (72-90\%) (Geomini et al., 2009). Considering the review and studies from Turkey our results were compatible. In our postmenopausal women, the RMI 2 (cut-off:200) yielded the sensitivity $88.6 \%$ and specificity of $80.0 \%$ with Kappa 0.667 . In premenopausal women , the RMI 2 (cut off:200) yielded the sensitivity $60.9 \%$ and specificity of $91.0 \%$ [AUC:0.89, CI:0.85-0.94], with Kappa 0.400 . Finally, in our study results of a general population and postmenopausal women were compatible with the literature, but an agreement of test was fair in premenopausal women.

Tingulastad et al. (1999) defined RMI 3 with cut off 200 , the sensitivity of $71 \%$ and specificity of $92 \%$. Bailey et al. investigated 182 patients. Malignancy Risk Index had 78.9, 70, 91.6 and 100\% sensitivity in Stage 1,2,3,4 ovarian cancers respectively. In all stages, RMI 3 had $87.4 \%$ sensitivity. (Bailey et al., 2006). In our study, the RMI 3 in a general population (cut off:200, kappa: 0.609) yielded a sensitivity of $76.1 \%$ and specificity of $90.6 \%$. In postmenopausal women, the RMI 3 (Kappa:0.711) formula had a sensitivity $84.0 \%$ and specificity of 87.7\%. The RMI 3 (cut off:200) method had a kappa 
value (0.409), sensitivity $60.9 \%$ and specificity of $91.8 \%$ in premenopausal women. The agreement of test and sensitivity was low in premenopausal women.

Yamamoto et al. (2009) reported 91\% specificity and $75 \%$ sensitivity for RMI 4. In two similar studies of Yavuzcan et al. and Ozun Ozbay et al. conducted in Turkey, the best performance for RMI 4 reported as 400. (with $91 \%$ specificity,75\% sensitivity and $92.4 \%$ specificity,67.4\% sensitivity, respectively) (Yavuzcan et al., 2013; Ozun Ozbay et al. 2015). In our study, The RMI 4 (cut off:336) had a sensitivity $76.1 \%$ and specificity of 88.6\% [AUC:0.90, CI: 0.85-0.94]. Although the interagreement validity of RMI 4 in a general population and postmenopausal women were moderate, the results of premenopausal women were fair.

The studies from Turkey shows similar results in the general population with sensitivity varies. In our study for the general population; RMI 1,2,3 with the cut-off value 200 and RMI 4 at the cut off 336 gave best sensitivity and specificity. RMI 1 was found to be a highly accurate test for a general population.

Few studies in the literature studies pre and postmenopausal women individually. Terzic et al. studied RMI 1 predictive values for pre and postmenopausal women (Terzic et al., 2013). At $83 \%$ sensitivity, in premenopausal women RMI had $80.31 \%$ specificity; in postmenopausal women RMI had $68.18 \%$ specificity. In our study, we had better results in the postmenopausal group like Sayasneh et al. (2013). Sayasneh et al. showed that; the RMI at a cutoff level of 200 yielded the sensitivity of $72 \%$, the specificity of 94\% [AUC:0.90, CI:0.83-0.94] in the general population. At the same cutoff value of RMI, premenopausal group yielded the sensitivity of 54\%, the specificity of 96\% [AUC:0.83, CI:0.67-0.92]. Postmenopausal group yielded the sensitivity of $83 \%$, specificity of $89 \%$ [AUC:0.92, CI:0.83-0.96]. In our study, postmenopausal women RMI 1 and 3 had the best performance with 200 cut off yielded $84 \%$ sensitivity and $87.7 \%$ specificity. In premenopausal women, RMI 1 was the most reliable test in terms of area under the curve, and for cut off 200 yielded $60.9 \%$ sensitivity and $92.5 \%$ specificity. A recent systemic review and meta-analysis by Kaijser et al. studied 19 different prediction models on 26438 adnexal masses included in this study (Kaijser et al., 2013). RMI was the most frequently validated model. Pre and postmenopausal status were also compared and RMI 1 (using a cut-off of 200) showed sensitivity 0.44 [95\%, CI:0.28-0.62] , specificity 0.95 [95\%, CI:0.90$0.97]$ in premenopausal women; sensitivity 0.79 [95\%, CI:0.72-0.85] , specificity 0.90 [95\%, CI:0.84-0.94] in postmenopausal women. . In this review Kaijser et al. (2014) suggests using IOTA Simple rules or the LR model (International Ovarian Tumor Analysis) especially in reproductive age women. Our results were similar to Kaijser et al. (2014) that RMI 1 had more diagnostic value in postmenopausal women and test performance was not good in premenopausal women

The main limitation of the study was its retrospective design. The major strength of our search for previous papers was a calculation of premenopausal and postmenopausal women separately. The difference in sensitivity rates may be attributed to the heterogeneity of ovarian cancers and prevalence of the disease. Such as sensitivity of a test decreased when borderline and nonepithelial tumor incidence increase.

In conclusion, In our study, RMI 1 in the general population and RMI 1 \& RMI 3 in postmenopausal women had the best performance in discriminating between benign and malignant in women with adnexal masses preoperatively. The performance of RMI algorithms in borderline tumors and premenopausal women was moderate or fair. Our study confirms the effectiveness of RMI algorithms and universally accepted cut-off values in clinical practice for the identification and subsequent referral to accurate centers in postmenopausal women. We need more sensitive tests for borderline ovarian tumors and premenopausal women.

\section{References}

Abdulrahman Jr.GO, McKnight L, Singh KL (2014). The risk of malignancy index (RMI) in women with adnexal masses in Wales. Taiwanese Journal Obs Gyn, 53, 376-81.

Aggarwal P, Kehoe S (2010). Serum tumour markers in gynaecological cancers. Maturitas, 67, 46-53.

Akturk E, Karaca RE, Alanbay I, et al (2011). Comparison of four malignancy risk indices in the detection of malignant ovarian masses. J Gynecol Oncol, 22,177-82.

Andersen ES, Knudsen A, Rix P, et al (2003). Risk of malignancy index in the preoperative evaluation of patients with adnexal masses. Gynecol Oncol, 90, 109-12.

Arun-Muthuvel V, Java V (2014). Pre-operative evaluation of ovarian tumors by risk of malignancy index , CA 125 and ultrasound. Asian Pac J Cancer Pre, 15, 2929-32.

Ashrafganggooei T, Rezaeezadeh M (2011). Risk of malignancy index in preoperative evaluation of pelvic masses. Asian Pac J Cancer Prev, 12, 1727-30.

Bailey J, Tailor A, Naik R, et al (2006). Risk of malignancy index for referral of ovarian cancer cases to a tertiary center: does it identify the correct cases? Int J Gynecol Cancer, 16, 30-4.

Clarke S, Grimshaw R, Rittenberg P, et al (2009) Risk of Malingnacy Index in the Evaluation of patients with adnexal masses. J Obstet Gynaecol Can, 3, 440-5.

Curtin JP (1994).Management of the adnexal mass. Gynecol Oncol, 55, 42-6.

Eisenkop SM, Spirtos NM, Montag TW, et al (1991). The impact of subspeciality on the management of advanced ovarian cancer. Gynecol Oncol, 47, 203-9.

Elit L, Bondy SJ, Paszat L, et al (2002). Outcomes in surgery for ovarian cancer, Gynecol Oncol, 87, 260-7.

Enakpene CA, Omigbodun AO, Goecke TW, et al (2009) Preoperative evaluation and triage of women with suspicious adnexal masses using risk of malignancy index. J Obstet Gynaecol, 35, 131-8.

Geomini P, Kruitwagen R, Bremer GL, et al (2009). The accuracy of risk scores in predicting ovarian malignancy: a systematic review. Obstet Gynecol, 113, 384-94.

Gibbs RS, Karlan BY, Haney AF, Nygaard I (2010). Danfort's Obstetrics and Gynecology $10^{\text {th }}$ ed.1061.

Jacobs I, Oram D, Fairbanks J, et al (1990). A risk of malignancy incorporating CA 125 , ultrasound and menopausal status fort he accurate preoperative diagnosis of ovarian cancer. Br J Obstet Gynecol, 97, 922-9.

Kaijser J, Sayasneh A, Van Hoorde K, et al (2014). Presurgical diagnosis of adnexal tumours using mathematical models and scoring systems: a systematic review and meta-analysis. 
Predictive Values of Malignancy Risk Indices for Ovarian Masses in Premenopausal and Postmenopausal Women

Human Reproductive Update, 20, 449-62.

Kehoe S, Powell J, Wilson S, et al (1994). The influence of the operating surgeons specialisation on patient survival in ovarian carcinoma. Int J Cancer, 70, 1014-7.

Moolthiya W, Yuenyao P (2009). The Risk of Malignancy Index (RMI) in Diagnosis of Ovarian Malignancy. Asian Pac J Cancer Prev, 10, 865-68.

Morgante G, la Marca A, Ditto A, et al (1999). Comparison of two malignancy risk indices based on serum CA125, ultrasound score and menopausal status in the diagnosis of ovarian masses. Br J Obstet Gynecol, 106, 524-7.

National Institutes of Health Consensus Development Conference Statement (1994). Ovarian cancer: screening, treatment, and follow-up. Gynecol Oncol, 55, 4-14.

NICE Clinical Guidelines (2011). CG122. Ovarian cancer: the recognition and intial menagement of ovarian cancer . Issued: April.

Obeidat BR, Amarin ZO, Latimer JA, et al (2004). Risk of malignancy index in the preoperative evaluation of pelvic masses. Int J Gynaecol Obstet, 85, 255-8.

Ong C, Biswas A, Choolani M, et al (2013). Comparison of risk of malignancy indices in evaluating ovarian masses in a Southeast Asian population. Singapore Med J, 54 ,134-9.

Ozols RF, Rubin SC, Thomas GM, et al (2005). Epithelial ovarian cancer. In: Hoskins WJ, Perez CA, Young R, Barakat R, editors. Principles and practice of gynecologic oncology. $4^{\text {th }}$ ed. Philadelphia, PA:Lippincott Williams and Wilkins, 895-987.

Ozun Ozbay P, Ekinci T, Demir Caltekin M, et al (2015). Comparative evaluation of the risk of malignancy index scoring system (1-4) used in differential diagnosis of adnexal masses. Asian Pac J Cancer Prev, 16, 345-9.

Prat J (2014). FIGO staging classification for cancer of the ovary, fallopian tube, and peritoneum.FIGO committee on gynecologic oncology. Int J Gynaecol Obstet, 124, 1-5.

RCOG guideline:ovarian cysts in postmenopausal women. October 2003.(Green top 34).

Sayasneh A, Wynants L, Preisler J, et al (2013) T. Multicentre external validation of IOTA prediction models and RMI by operators with varied training. Br J Cancer, 108, 2448-54.

Siegel R, Ma J, Zou Z, et al (2014). Cancer Statistics, 2014. CA Cancer J Clin, 64, 9-29.

Su Z, Graybill WS, Zhu Y (2013). Detection and monitoring of ovarian cancer. Clin Chim Acta, 415, 341-5.

Simsek HS, Tokmak A, Ozgu E, et al (2014) Role of risk of malignacy index in clinical approach to adnexal masses. Asian Pac J Cancer Prev, 15, 7793-97.

Terzic M, Dotlic J, Likic I, et al (2013). Risk of malignancy index validity assessment in premenopausal and postmenopausal women with adnexal tumors. Taiwan J Obstet Gynecol, 52, 253-7.

The role of the generalist obstetrician-gynecologist in the early detection of ovarian cancer (2002). ACOG Committee Opinion No. 280. american college of obstetricians and gynecologists. Obstet Gynecol, 100, 1413-6.

Tingulstad S, Hagen B, Skjeldestad FE, et al (1996). Evaluation of risk of malignancy index based on serum CA 125 , ultrasound findings and menopausal status in the preoperative diagnosis of pelvic masses. Br J Obstet Gynecol, 102, 826-31.

Tingulstad S, Hagen B, Skjeldestad FE, et al (1999) The risk of malignancy index to evaluate potential ovarian cancers in ocal hospitals. Br J Obstet Gynecol, 93, 448-52.

Van den Akker PA, Aalders AL, Snijders MP, et al (2009) Evaluation of the risk of malignancy index in daily clinical management of adnexal masses. Gynecol Oncol, 116,384-8.

Van Gorp T, Veldman J, Van Calster B, et al (2012). Subjective assessment by ultrasound is superior to the risk of malignancy index (RMI) or the rsk of ovarian malignancy algorithm (ROMA) in discriminating benign from malignant adnexal masses. Eur J Cancer, 48, 1649-56.

Van Trappen PO, Rufford BD, Mills TD, et al (2007) Differential diagnosis of adnexal masses : risk of malignancy index, ultrasonography, magnetic resonance, and radioimmunoscintigraphy. Int J Gynecol Cancer, 17, 61-7.

Winarto H, Laihad BJ, Nuranna L (2014).Sonographic morphology scores (SMS) for differentiation between benign and malignant adnexal masses. Asian Pac J Cancer, 7, 407-10.

Yamamoto Y, Yamada R, Oguri H, et al (2009). Comparison of four malignancy risk indices in the preoperative evaluation of patients with pelvic masses. Eur J Obstet Gynecol Reprod Biol, 144, 163-7.

Yavuzcan A, Caglar M, Ozgu E, et al (2013). Should cutoff values of the risk of malignancy index be changed for evaluation of adnexal masses in Asian and Pacific populations? Asian Pac J Cancer Prev, 14, 5455-9.

Yazbek J, Aslam S, Tailor A, et al (2006). A comparative study of the risk of malignancy index and the ovarian crescent sign fort he diagnosis of invasive ovarian cancer. Ultrasound Obstet Gynecol, 28, 320-4.

Yesilyurt H, Tokmak A, Güzel Aİ, et al (2014). Parameters for predicting granulosa cell tumor of ovary a single center retrospective comparative study. Asian Pac J Cancer Prev, 15, 8447-50. 